Voix et Images

voixetimages

\title{
Qui a peur de l'intellectuel en 1987?
}

\section{Pierre Milot}

Volume 12, numéro 3 (36), printemps 1987

Yves Beauchemin

URI : https://id.erudit.org/iderudit/200666ar

DOI : https://doi.org/10.7202/200666ar

Aller au sommaire du numéro

Éditeur(s)

Université du Québec à Montréal

ISSN

0318-9201 (imprimé)

1705-933X (numérique)

Découvrir la revue

Citer cet article

Milot, P. (1987). Qui a peur de l'intellectuel en 1987? Voix et Images, 12(3),

530-534. https://doi.org/10.7202/200666ar

Ce document est protégé par la loi sur le droit d'auteur. L'utilisation des services d'Érudit (y compris la reproduction) est assujettie à sa politique d'utilisation que vous pouvez consulter en ligne.

https://apropos.erudit.org/fr/usagers/politique-dutilisation/
Cet article est diffusé et préservé par Érudit.

Érudit est un consortium interuniversitaire sans but lucratif composé de l’Université de Montréal, l'Université Laval et l'Université du Québec à Montréal. Il a pour mission la promotion et la valorisation de la recherche. https://www.erudit.org/fr/ 


\section{Qui a peur de l'intellectuel en 1987?}

\section{par Pierre Milot, Université du Québec à Montréal}

"Qui a peur de l'écrivain?», se demandaient les signataires du recueil collectif publié par les Herbes rouges en 1984'. D'autres écrivains, serait-on tenté de répondre à la lecture de l'échange épistolaire consigné par Hugues Corriveau et Normand de Bellefeuille dans A double sens («Echanges sur quelques pratiques modernes") et publié chez le même éditeur ${ }^{2}$. Rédigé entre mars 1984 et août 1985 (soit au beau milieu de cette polémique entre la modernité copyright et la question du religieux), ce livre écrit à partir d'une quarantaine de lettres que se sont adressés les deux écrivains ne fait que contourner le symptôme du conflit, il ne l'assume pas. Non pas que cet ouvrage sur les "pratiques modernes" aurait dû constituer d'un seul bloc une réponse aux invectives lancées par François Charron et André Beaudet (les 
deux auteurs du recueil dont les propos contenaient la plus forte dose de violence symbolique), mais l'on aurait pu s'attendre à un débat articulé plutôt qu'à une tractation qui frôle la dénégation.

\section{Écrivain et/ou intellectuel en 1987 ?}

Si je jette les dés de cette façon, à partir d'une polémique datée, c'est qu'un enjeu s'est perdu en cours de route, c'est-à-dire depuis la parution de ce fameux numéro de la Nouvelle Barre du jour posant la question litigieuse: "Intellectuel/le en 1984?». Dans son texte sur «la pensée en panne» et sur "l'urgence du réel», Normand de Bellefeuille semblait s'inquiéter de certains tournants (et retournements) de l'Histoire (la majuscule est de lui): l'intellectuel/le québécois/se risque présentement de redevenir un être sans substance, sans parole spécifique (p. 52). Et un peu plus loin: Encore plus que les lieux d'action si prestement liquidés, peut-être est-ce cette idée d'une solidarité intellectuelle qui fait ici défaut (p.53). Et pour bien marquer le coup de cette posture intellectuelle, deux figures référentielles: le Scribe de Régis Debray et les Questions de principes de Bernard-Henri Lévy, citations à l'appui. Les cibles visées sont on ne peut plus évidentes: Ainsi nous assistons, depuis peu, dans le milieu intellectuel, à une inquiétante recrudescence de certaines vieilles tendances contre-culturelles: théories de "l'aura» et délire zodiacal, énergies diverses (l'énergie comme "relève» à l'idéologie) et gouroumanie galopante, égyptophilie, néo-mysticismes... bref, une étonnante réactualisation de tous ces discours de l'indicible, de l'essentiel, de l'innommable, du Mystère (p. 48). En février 1984, lors du Colloque de la Nouvelle Barre du Jour sur la modernité ${ }^{3}$, Hugues Corriveau fourbira les armes contre un prétentieux retour à Dieu et à d'autre mythologies, égyptiennes et mexicaines, alors que la modernité des années 1980 devrait s'occuper du réel et du présent (p. 86). Même s'ils ne sont pas nommés, les Charron et Beaudet (mais aussi les Beausoleil et Villemaire) sont ici incriminés.

Ce n'est donc pas un hasard si les deux premiers lanceront un pamphlet pour répondre au réquisitoire de Corriveau et de Bellefeuille. Ils poseront le différend en termes d'enrégimentement de l'écrivain dans la Théorie et le Réel, et se proposeront de critiquer la conception moderne et réeliste de la littérature que certains des participants à ce numéro de la NBJ voudraient voir triompher (p. 2). Contre les accusations de "retour au mysticisme", on réplique par des énoncés incriminants du type: les intellectuels qui montent la garde du Réel. De plus, le paradigme de l'intertextualité y est utilisé comme pièce à conviction contre cette modernité obligatoirement branchée sur une connaissance des recherches dans le domaine du texte en France (p. 25). Ce qui n'empêche pas Beaudet de convoquer les figures de Lacan et de Sollers pour défendre sa propre cause, mais qui permettra à Charron de servir une dure leçon de contradiction logique à ses concurrents: nous relancer des citations de Regis Debray ou Bernard-Henri Lévy, faisant se côtoyer dans la plus belle entente ces deux frères ennemis (p. 17-18). Ainsi, derrière l'enjeu esthétique de la modernité et du sacré, se profilait un autre enjeu, social celui-là, concernant la posture intellectuelle de l'écrivain dans les années 1980. Qu'est donc devenu cet enjeu dans $\bar{A}$ double sens? 


\section{La guerre contre les saintes écritures n'aura pas lieu}

Comme je l'avais déjà mentionné ailleurs ${ }^{4}$, parler d'une polémique entre la NBJ et les Herbes rouges induisait une polarité nominale mal appropriée (p. 126), puisque les Corriveau et de Bellefeuille avaient pour ainsi dire publié toutes leurs ouvres aux Herbes rouges. Pourtant, cet effet de concurrence produit par le débat n'est pas le résultat d'une quelconque confusion entretenue par l'institution littéraire, comme le laissait entendre dernièrement André Beaudet dans une entrevue de la revue Moebius, où il feint de s'interroger sur Qu'est-ce qui intéresse l'institution littéraire là-dedans? ${ }^{5}$. En fait, les indicateurs de polarité éditoriale avaient été posés par les écrivains incriminés, de part et d'autre: certains écrivains québécois regroupés autour de revues comme Les Herbes rouges ${ }^{6}$ pour les premiers, et Groupe qu'on retrouve attaché au magazine Spirale et à la revue La Nouvelle Barre du jour?, pour les seconds. S'il y a eu ambiguité, elle ne provient nullement de l'institution littéraire (le "discours institutionnel» dirait Beaudet), mais des écrivains eux-mêmes. Et d'ailleurs, l'effet institutionnel de ce conflit n'a pas été sans procurer un profit symbolique, tant aux deux éditeurs qu'aux écrivains des deux maisons, qui ont vu leurs titres et leurs noms prendre de l'expansion dans l'espace publicitaire du champ littéraire.

Corriveau et de Bellefeuille ont donc décidé de ne pas en remettre dans leur essai sur «les pratiques modernes» de l'écriture telle qu'ils la produisent aux Herbes rouges... et à la NBJ (dont de Bellefeuille est secrétaire de rédaction). Bien sûr, comme je l'ai mentionné précédemment, on en sent les stigmates mais le symptôme en est contourné par des relations textuelles d'un autre ordre. Un ordre de procédés rhétoriques axés autour de diverses isotopies: la modernité, le désir, la forme, le vide, etc. On y sent le métier, la technique d'écriture, à chaque page, d'une lettre à l'autre. Mais s'agit-il vraiment d'un échange rigoureux (comme le proclame la maquette de couverture), et que peut bien signifier cette conscience des limites de toute volonté théorique que l'on nous sert sur le mode préventif, alors que précisément la théorie est en perte de vitesse dans ce livre de poses textuelles? La leçon de l'écrivain à l'intellectuel a-t-elle été si dure que ce dernier a décidé, en tant qu'écrivain, de taire ce qui faisait parler l'intellectuel? Ou bien la cause était-elle si légèrement soutenue qu'un seul assaut ait pu décharger cette pensée de sa tension?

\section{Une esthétique sans éthique}

Avant d'aller plus loin, et pour que mes propos soient compris dans un sens et non dans un autre, je tiens à préciser que je ne cherche pas ici à intenter un procès pour préciosité formaliste et absence d'engagement politique, sachant fort bien ce que le discours du réalisme littéraire (et ses champs d'application) peut comporter d'illusionnisme social. Ce qui se dépense dans le conflit récurrent qui oppose les esthétiques du formalisme et du réalisme relève de l'économie des échanges littéraires: et le travail critique, au sens où je l'entends, consiste à objectiver les mécanismes de régulation de cette économie de pratiques concurrentes, non à investir dans une entreprise esthétique plutôt que dans une autre. La sociologie littéraire est à ce prix. 
Dans l'avant-dernière lettre de l'échange épistolaire, Corriveau écrit: Pourtant, quarante textes plus loin, la séduction opère suffisamment pour que (comme moi, dans ce livre) tu cites aussi tes propres textes... conviant le lecteur à tomber sous le charme (p. 210). Mais le lecteur qui laisse tomber le livre, après le dernier texte, se sent-il séduit ou réduit? Réduit à constater «l'évidence formelle» de ces rapports textuels entre deux écrivains modernes «dans l'instant du papier"? Y aurait-il du texte qui se produirait à travers nous? (p. 107) se demande Corriveau (ici le lecteur doit cocher Lacan: L' inconscient, ça parle). Mais ailleurs, les repères sont nommés, qui plus est, accrédités: il s'agit bien au contraire de reconnaître au mécanisme sa part de responsabilité dans la production du désirant et du pulsionnel, ce qui devrait aussi, depuis Deleuze, être définitivement acquis (p. 166). Depuis Deleuze? Une responsabilité du mécanisme? Du définitivement acquis? Leçon sur la leçon: de Bellefeuille sait-il que l'inconscient lacanien et l'inconscient deleuzien ne relèvent pas d'une même problématique du désir? Et que s'il est possible de faire se rencontrer une machine à coudre et un parapluie sur une planche à repasser dans un texte de fiction, il est beaucoup moins rigoureux d'amalgamer le structuralisme et le post-structuralisme dans un essai théorique. Et quand, en plus, on rajoute à cet agrégat discursif le post-modernisme de Guy Scarpetta (p. 197-200), le lecteur comprend mieux ce que signifiait la formule concernant les limites de toute volonté théorique. Car Lacan, Deleuze et Scarpetta dans le même boudoir, ce n'est plus de l'impureté esthétique, c'est de la confusion conceptuelle.

Et le concept de modernité dans tout cela? Il y est introduit par une référence à Introduction à la modernité d'Henri Lefebvre ${ }^{8}$ curieusement revisité pour une brève incursion dans l'avant-garde des années 1970 au Québec, époque où la modernité québécoise se serait révélée en littérature: Plusieurs écrivains de la modernité naissante risquent alors une inscription de leur pratique d'écriture à même une pratique révolutionnaire au sens premier du terme (p. 21). En somme, la modernité québécoise en littérature se mesurerait à l'aune de la "modernité poétique» des Herbes rouges et de la Barre du jour. On comprend pourquoi les auteurs se sentent plus à l'aise dans le champ des formes que dans le champ des études littéraires.

Des formes et des études, il en est d'ailleurs question dans l'ironique «Lettre scolaire" (p. 40-44) que de Bellefeuille adresse à "l'élève Corriveau", et qui donnera lieu à un exercice de style théorique rempli d'émotions (p. 45-64). Le "débat" porte sur la suppression de la lettre "e» dans un fragment de Cold Cuts: l'un y perçoit la coupe du Père, l'autre y débusque la pulsion de la Mère. Figures rythmiques qui feront s'exclamer de Bellefeuille: nous voguons en pleine théorie! ( $\mathrm{p}$. 74). Pour ne pas rester en reste, Corriveau soumettra à la discussion une output sheet d'un texte de France Théoret (la Marche) dont l'ordinateur a chiffré un certain nombre de récurrences lexicales et syntaxiques.

On le voit, pour Corriveau et de Bellefeuille, l'Histoire a perdu sa majuscule (suite aux remontrances de leurs pairs) et la "pensée en panne" a pris le virage technologique. Se pourrait-il que dans un prochain tournant (ou retournement) «l'urgence du réel» refasse surface et que la problématique de 
l'intellectuel redevienne une question à débattre? Mais en cette fin des années 1980 (arrimée à une fin de siècle), qui donc a peur de l'intellectuel? Assurément, les écrivains d'Ā double sens. Comme c'est le cas pour toute esthétique sans éthique.

1. Les Herbes rouges no $123-124$, $1^{\text {er }}$ trimestre 1984 . Voir le liminaire de François Charron.

2. Editions Les Herbes rouges, 1986.

3. Vouloir la fiction. "Copyright la modernité», Éditions de la NBJ, 1984. Voir le «reportage" de Jean Royer: "Ä Montréal, la modernité».

4. "L'avant-garde: processus institutionnel et conflits de légitimité» in L'institution littéraire (sous la direction de Maurice Lemire), Institut Québécois de Recherche sur la Culture, 1986, p. 115-128.

5. Beaudet y déclare: Je viens de lire la livraison du printemps de Voix et images et les actes du colloque sur L'institution littéraire. C'est inoui! Au moins cinq articles sont reliés, de près ou de loin, au procès de la modernité, aux tenants et aboutissants d'une polémique qui a défrayé la chronique littéraire. in Moebius, no 30, automne 1986, p. 14. Ce qui est «inouï), c'est que Beaudet, principal promoteur du conflit modernité/sacré, feigne de s'étonner d'une stratégie de relation publique qu'il a lui-même opérationnalisée.

6. Cf. «Intellectuel/le en 1984?», La NBJ, no 130-131, octobre 1983, p. 146, note 16.

7. Cf. "Qui a peur de l'écrivain?" op. cit., p. 8, note 1.

8. Peut-on sérieusement vouloir traiter des conditions sociales de la modernité sans passer par le débat Habermas-Lyotard sur les rapports entre modernité et postmodernité? Et discuter d'avant-garde sans passer par Bürger? Bien sûr, ce n'est pas un passage obligatoire, mais tout de même.

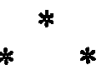

